\title{
Modelling the role of the basement membrane beneath a growing epithelial monolayer
}

\author{
Sara-Jane Dunn ${ }^{\mathrm{a}, *}$, Alexander G. Fletcher ${ }^{\mathrm{b}, \mathrm{c}}, \mathrm{S}$. Jonathan Chapman ${ }^{\mathrm{b}}$, \\ David J. Gavaghan ${ }^{\mathrm{a}, \mathrm{c}}$, James M. Osborne ${ }^{\mathrm{a}, \mathrm{c}}$ \\ a Department of Computer Science, University of Oxford, Wolfson Building, Parks Road, Oxford, OX1 3QD, UK \\ ${ }^{\mathrm{b}}$ Mathematical Institute, 24-29 St. Giles', Oxford, OX1 3LB, UK

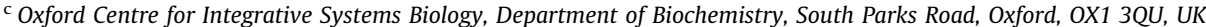

\section{A R T I C L E I N F O}

\section{Article history:}

Received 4 January 2011

Received in revised form

12 November 2011

Accepted 13 December 2011

Available online 22 December 2011

Keywords:

Curvature

Epithelia

Basement membrane

\begin{abstract}
A B S T R A C T
The role of the basement membrane is vital in maintaining the integrity and structure of an epithelial layer, acting as both a mechanical support and forming the physical interface between epithelial cells and the surrounding connective tissue. The function of this membrane is explored here in the context of a growing epithelial monolayer, defined such that the epithelial cells divide and migrate along a deformable substrate. A discrete, off-lattice cell-centre modelling approach is undertaken, which permits definition of a basement membrane component, separating the epithelial cells from the tissue stroma whilst responding to forces from both that arise due to cell division, migration and apoptosis. This model is applicable to a range of biological epithelia, including the self-renewing interfollicular epidermis, the olfactory epithelium and the intestinal crypts of Lieberkühn, to inform response and recovery of such tissues following injury. Model simulations show that homeostasis of the growing monolayer can be achieved and sustained, and the necessary balance of interactive cell forces, cell migration and cell death is presented. This work is proposed as a novel extension to the body of discrete models of biological epithelia, permitting investigation of the growth and migration of epithelial cells in a deformable environment.
\end{abstract}

(c) 2011 Elsevier Ltd. All rights reserved.

\section{Introduction, background and motivation}

Epithelial cell sheets pervade the body, covering all of the internal organs and cavities, as well as forming the skin. The high turnover of cells within these layers increases vulnerability to carcinogenesis, and indeed the majority of cancers that develop arise from epithelial cells, known as carcinomas, and this provokes interest in the function and role of particular epithelia under healthy and unhealthy conditions. One such example is colorectal cancer (CRC), which is thought to originate via genetic alterations that cause disruption to the cellular dynamics of the crypts of Lieberkühn, test-tube shaped glands located in the intestine, which are lined with a monolayer of epithelial cells. A delicate balance of cell division, migration and death is coordinated in the crypts to renew the epithelial layer every few days (Näthke, 2004; Shanmugathasan and Jothy, 2000). CRC develops as a consequence of epithelial cells that accumulate genetic mutations which alter migration patterns, increase proliferation, provide

\footnotetext{
* Corresponding author. Tel.: +44 1865 273838; fax: +44 1865273839

E-mail addresses: sara-jane.dunn@microsoft.com (S.-J. Dunn),

fletcher@maths.ox.ac.uk (A.G. Fletcher), chapman@maths.ox.ac.uk (S.J. Chapman), david.gavaghan@cs.ox.ac.uk (D.J. Gavaghan),

james.osborne@cs.ox.ac.uk (J.M. Osborne).
}

resistance to apoptosis cues, or disrupt cell adhesion, acquiring the ability to persist and multiply in the crypts.

In the same way that the intestinal epithelial layer is maintained by the cells that line the crypts, skin tissue itself is replenished by a population of stem and transit-amplifying cells (Clayton et al., 2007). The hair follicles in the skin are surrounded by the interfollicular epidermis, which comprises a basal layer of proliferating cells that produce cells committed to differentiation, which migrate towards the upper cell layers.

A third example is that of the olfactory epithelium (OE), self-renewing neural tissue lining the nasal cavity, which is responsible for transmitting odour information to the brain. This particular epithelium sits above glands similar to the crypts of Lieberkühn, known as Bowman glands. Research into the OE has concentrated on understanding the relationship between growth control and cell lineage - like the crypt, the OE maintains a stable size through continuous replacement of dying cells, such as the primary olfactory receptor neurons (ORNs), which can be killed by pathogens entering the nose (Duggan and Ngai, 2007). Research is seeking to define a population of stem cells which generates all of the cell lineages, and which is responsible for replenishing the epithelium following injury. Direct parallels can be drawn to the cell lineage model in the crypt, and the strict control exerted to maintain a stable population throughout regular cell turnover. 
Indeed repair following injury to the airway epithelium is of interest in light of the mechanical strain imposed during the cyclic deformation throughout respiration (Savla et al., 2004).

To achieve accurate, reliable predictions of the breakdown of epithelial structures that occurs at the onset of injury, a model must link processes occurring at the subcellular, cellular and tissue levels. Such a model must also take into account the geometry of the specific epithelium, which may be flat or folded (Smallwood, 2009), and the tissue structure itself. In this way, a model allows tissue-level behaviour to emerge as a consequence of cell-level behaviour. This work specifically addresses the role of the basement membrane, the primary contact of the epithelium to the extracellular matrix. By proposing a model for this important component of the tissue structure, it is possible to investigate growth of an epithelial layer in a deformable domain, and this can subsequently be applied to consider specific cases, such as the onset of carcinogenesis in the intestinal crypts.

Epithelial sheets consist of one or more layers of contiguous cells that are anchored to a basement membrane, an extracellular layer which separates the epithelium from the underlying connective tissue whilst providing essential mechanical support (Alberts et al., 2002; Wright and Alison, 1984). The basement membrane is comprised of a basal lamina and a reticular lamina; the former is a thin mat composed primarily of Type IV collagen that is attached to the basal side of the epithelial cells, and also by collagen fibrils to the reticular lamina, which is attached to the connective tissue. Evidence suggests that the interactions between the epithelium and basement membrane promote cell adhesion, induce expression of differentiated cell functions, initiate and maintain cell polarity, participate in the regulation of morphogenesis and promote cell migration (Trier et al., 1990).

Epithelial cells exhibit planar cell polarity, whereby the apical membrane of each cell points towards the lumen and, during symmetric division, the mitotic spindles align parallel to the tissue layer (Fleming et al., 2007). Consequently a cell places its daughter cell next to it within the plane of the epithelium. A form of programmed cell death, anoikis, is triggered when there is inadequate adhesion of the epithelial cells to the extracellular matrix (Frisch and Francis, 1994), with detachment inducing apoptosis for healthy cells (Bullen et al., 2006; Grossman, 2002). Functioning correctly, this maintains tissue homeostasis by restricting proliferation to the epithelium, thereby averting dysplasia, and by preventing cells from re-attaching in another location and resuming growth.

A number of mathematical models exist that seek to describe specific aspects of the behaviour of epithelial cells, and epithelial cell sheets, from cell cycle models that govern mitosis of individual cells (Swat et al., 2004; Van Leeuwen et al., 2009), to cellular automata and lattice-free mechanical models of cell proliferation and migration (Honda et al., 2004; Loeffler et al., 1988; Meineke et al., 2001). However, as yet, a model has not been proposed which considers the role of the basement membrane and surrounding connective tissue in influencing cell dynamics. This prevents the use of such models to realistically examine the tissue-level effects of abnormal cell behaviour.

For the case of the intestinal crypts, models do exist, however, that simulate crypt buckling and fission. Edwards and Chapman (2007) present a continuum representation of the crypt, modelled as a growing beam, whilst Drasdo and Loeffler (2001) apply an off-lattice cellular automata model to a U-shaped chain of deformable circles. These models commonly assign a bending stiffness to the layer, and predict that buckling will occur if growth by cell division is not adequately matched by this force. The former generalises cell division events and so does not permit the inclusion of cell cycle models that govern mitosis, and neither take into account the deformation of the surrounding tissue stroma. In addition to addressing these two points, to improve on this basis of existing work, and because of the coupling that exists between events at the genetic level through to the tissue level, a multi-scale model must be employed to model epithelial layers. Further, the roles of migration, cell-cell and cell-matrix adhesion must be considered.

The role of the basement membrane and supportive connective tissue is investigated here by following the evolution of a monolayer of proliferating cells that is constrained to lie on a bed of non-proliferating stromal cells, which approximate the connective tissue. Once this model is understood in a simple geometric framework, it can be incorporated and extended to consider specific biological epithelia.

The remainder of this paper is composed as follows. The theoretical framework of the model is described in Section 2, which details existing methods that are implemented and introduces the new method that is defined to model the basement membrane. The computational framework used to implement simulations is also introduced here. Results are presented in Section 3, and subsequently discussed in Section 4. These results inform future work, which will focus on the application of this basement membrane modelling approach to specific epithelia, in particular, the crypts of Lieberkühn.

\section{Methods}

The components of the model are now described in detail. These include existing methods that define cell connectivity and prescribe cell mechanics, as well as new methods that govern division and death, and importantly, model the role of the basement membrane.

Cell centres are defined as nodes which evolve spatially according to an off-lattice definition of cell-cell mechanics (Meineke et al., 2001; Van Leeuwen et al., 2009). As such, spatial connectivity is determined by a Delaunay triangulation of cell centres, and the corresponding cell shapes are subsequently defined by the dual Voronoi tessellation, which has been shown to produce realistic polygonal cell shapes (Honda, 1983). An example of this triangulation and tessellation is illustrated in Fig. 1.

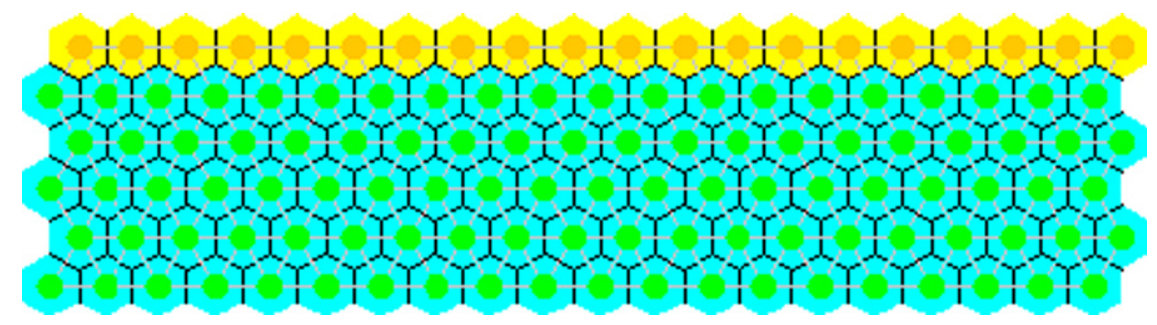

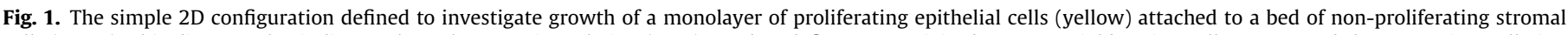

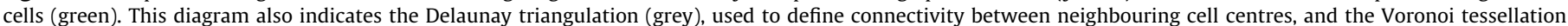

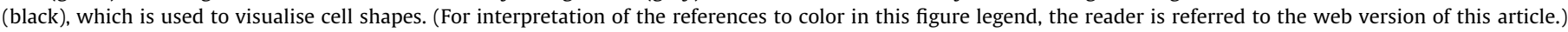


The simple geometry considered for this modelling work is also shown in Fig. 1, where a monolayer of proliferating cells (yellow) is prescribed to sit atop a bed of non-proliferating stromal cells (green). As such, the lumen is the space above the layer of proliferating cells, and the connective tissue is approximated as a collection of cells that are connected as described above. The structural properties of the stroma can be altered by varying the interactive cell forces, which in turn define the rigidity and density of the collection of cells.

The initial mesh is a block of 20 by 6 nodes. This is constructed using a simple, rectangular honeycomb mesh, surrounded by a layer of 'ghost nodes'. These nodes are labelled as ghosts because, whilst they experience a force due to real, neighbouring nodes, they do not exert any force in response. Such nodes have been implemented previously by Van Leeuwen et al. (2009). Without ghost nodes, the edges of the mesh do not form a convex hull, resulting in long connections between nodes at the edge which correspond to large spring forces. By including the extra layer of ghost nodes, the long connections are prevented, and only the ghost nodes have infinite tessellations. The simulation then follows only the real cells. The individual components of the model are now introduced.

\subsection{Cell-cell forces}

Interactive forces between cells are modelled as linear, overdamped springs that act along the edges of the triangulation; consequently, a steady-state distribution of cells is reached in the absence of proliferation. The total force acting on each cell is found by calculating the sum of the contributing forces from the springs that connect it to each neighbouring cell

$\mathbf{F}_{i}(t)=\mu \sum_{\forall j(t)} \hat{\mathbf{r}}_{i, j}(t)\left(s_{i, j}(t)-\left|\mathbf{r}_{i, j}(t)\right|\right)$

Here, $\mathbf{r}_{i, j}(t)$ is the vector from cell centre $i$ to $j, \hat{\mathbf{r}}_{i, j}(t)$ is the corresponding unit vector, $s_{i, j}(t)$ is the rest length of the spring, and $\mu$ is the spring constant.

By considering inertial terms as small compared with dissipative terms, the velocity of node $i$ is given by

$\eta \frac{d \mathbf{r}_{i}}{d t}=\mathbf{F}_{i}(t)$

where $\mathbf{r}_{i}$ the position of node $i$, and $\eta$ is a constant drag term, which represents general resistance analogous to motion in an aqueous medium. The system is evolved in small intervals, and cell positions are updated at each timestep using the Forward Euler method (Ascher and Petzold, 1998).

By modifying the spring constant, $\mu$, the magnitude of cell-cell adhesion or repulsion is varied. In reality, both epithelial cells and stromal cells are adhered to the basement membrane, but have no direct connections to each other. However, it is the case that the surrounding tissue implicitly provides support to the epithelial layer, and resists deformation and invasion. In order to invade the surrounding tissue stroma, epithelial cells must secrete digestive enzymes to first break through the basement membrane. To model this, the spring connections between epithelial and stromal cells are defined to exert a force only under repulsion. In this way, stromal cells do not pull at epithelial cells and the epithelial cells are tightly adhered to the basement membrane by applying a strong force, henceforth referred to as the basement membrane force, which is described at the end of this section. The spring constant applied between different types of cells can be varied, and values are chosen to maintain stability of the layer, as discussed in Section 3.

\subsection{Cell division}

The epithelial cells in the monolayer are proliferative, and divide according to a simple stochastic cell cycle model, with the duration of the cell cycle chosen for each individual cell from a $X \sim U(11,13)$ distribution (Alberts et al., 2002). The epithelial cells are assigned unlimited proliferative capacity, i.e. do not differentiate, for the purposes of this investigation, to consider a continuously growing monolayer. The stromal cells are defined to be terminally differentiated, and so do not divide.

To model the directed cell division that arises from planar cell polarity, each cell is instructed to divide according to the relative position of the nearest epithelial neighbours. Two daughter nodes are created in place of the original parent node, connected by a spring that lies parallel to the vector that connects the nearest neighbours of the parent node, and placed a small distance away $(0.05 s)$ on either side of the parent node position, where $s$ is the rest length of the spring between two fully mature cells, scaled to 1 cell width. The new connecting spring is assigned a rest length, $s_{i, j}(t)$, which increases linearly from $0.1 s$ to $s$ over the course of $\mathrm{M}$ phase $(1 \mathrm{~h})$, which models the growth of a cell before division occurs, at which point two new daughter cells are created. In the event that the monolayer is corrupted, which will occur if the layer buckles, it may arise that a dividing cell has more than two epithelial neighbours. In this situation, the spring that connects the two daughter nodes is assigned a randomly chosen axis.

\subsection{Boundary conditions and cell death}

Rigid boundary conditions are imposed on the vertical walls of the stroma, and the bottom row of nodes - those at the base of the tissue stroma - is held pinned. Anoikis is implemented in the top layer by defining a cell to undergo apoptosis should it lose all connections to the basement membrane, retaining only connections to other epithelial cells in the monolayer. This is determined using the Delaunay triangulation of the cell centres, and an example of two cells undergoing anoikis is shown in Fig. 2. Once a cell is apoptotic, it is removed from the simulation. Given that, by definition, the epithelial cells that this apoptotic cell was connected to are also connected to one another, this does not result in holes in the monolayer. Further, to model the migration of cells beyond the edges of the defined domain, epithelial cells

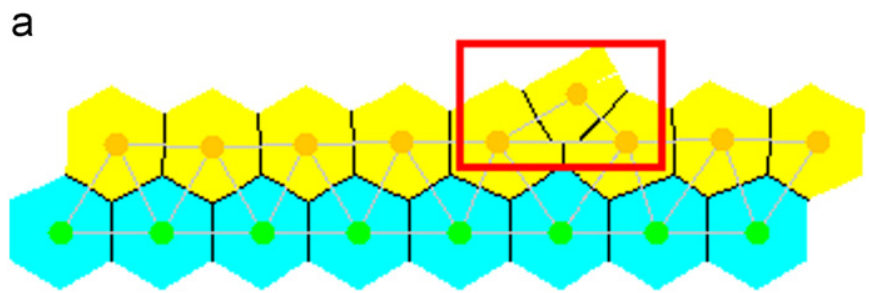

b

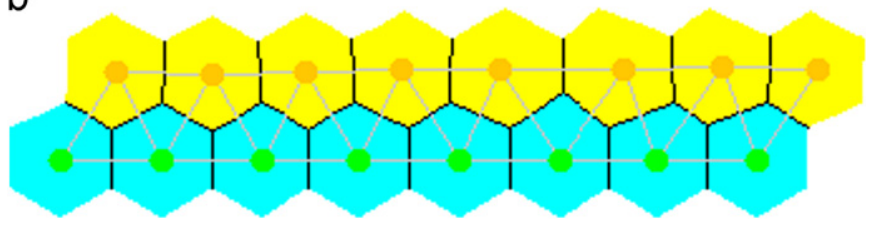

Fig. 2. An illustrative 'before and after' example of how the Delaunay triangulation is implemented to determine when a cell has lost contact with the underlying basement membrane. (a) An epithelial cell has moved up and out of the monolayer-this is contained in the rectangle, along with the two epithelial cells to which it has retained connections, as determined by the Delaunay triangulation. Hence this cell has lost contact with the basement membrane. (b) After anoikis has been applied, this cell is removed from the simulation and the cell centres are remeshed. 
that move beyond the ends of the vertical walls of stromal cells are removed from the simulation. These two mechanisms of cell removal are subsequently referred to as anoikis and sloughing respectively.

\subsection{Basement membrane force}

The Delaunay triangulation determines that each epithelial cell has at least one connection to the stromal cells surrounding the crypt (otherwise it would be removed from the simulation by anoikis), which are modelled as springs. The basement membrane, to which both epithelial and stromal cells are connected, is modelled as a piecewise linear curve that passes through the midpoints of these springs, as shown in Fig. 3(a), which magnifies a portion of the epithelial layer and the top layer of stromal cells. This membrane is not dependent on the Voronoi regions of the individual cells, which are used for visualisation purposes only.

Beneath a flat epithelial monolayer, the basement membrane itself will be flat, which for a smooth, continuous curve corresponds mathematically to a curvature of zero. As the basement membrane is modelled here as a piecewise linear interpolation of the spring midpoints, the discrete equivalent is sought for the curvature, which is hereafter referred to as the discrete curvature. A force is applied which is dependent on the local discrete curvature of the membrane, which acts to maintain a zero target curvature-from this point, this is referred to as the basement membrane force.

For each spring that connects an epithelial cell to a stromal cell, there will be a force acting along that spring according to the signed curvature of the membrane at the spring midpoint. The discrete curvature is calculated locally, using the Delaunay triangulation to identify which elements of the mesh are shared between an epithelial and stromal node pair, and the midpoints of the neighbouring springs are taken to form a piecewise linear curve defined by three points, as shown in Fig. 3(b). These points are then used to calculate the discrete curvature parametrically according to the calculation below, and the restoring force acts in proportion to this curvature and in the direction from the stromal cell centre towards the epithelial cell centre. The application of this force is similar to the stabilising bending force of Drasdo and Loeffler (2001), which is applied to correct deviation of a cell away from its spontaneous curvature. It is also equivalent to the bending stiffness considered in the continuum model of Edwards and Chapman (2007). However, these earlier models applied a bending stiffness directly to the epithelial monolayer, as distinct from modelling the basement membrane explicitly, as is the case here.

\subsection{Curvature calculation}

The definition of curvature for a smooth, continuous curve is as follows. Let $C$ be a curve defined parametrically by $\mathbf{r}(s)=(x(s), y(s))$, where $s$ is arc length. The signed curvature, $\kappa(s)$, at a point $P$ on $C$ measures the rate of change, $\mathbf{u}^{\prime}(s)$, of the unit tangent vector, $\mathbf{u}(s)$, at $P$. Now, $\mathbf{u}(s)=\mathbf{r}^{\prime}(s)$ and thus

$\kappa(s)=\mathbf{u}^{\prime}(s)=\mathbf{r}^{\prime \prime}(s)$.

In terms of the coordinates $x$ and $y$, this is

$\kappa(s)=\frac{x^{\prime} y^{\prime \prime}-y^{\prime} x^{\prime \prime}}{\left(x^{\prime 2}+y^{\prime 2}\right)^{3 / 2}}$,

where ' denotes $d / d s$. The sign of the curvature indicates in which direction the unit tangent vector rotates as a function of the parameter along the curve. If $\kappa>0$, then the unit tangent vector rotates anticlockwise, and conversely if $\kappa<0$, it rotates clockwise.

Applying this to the epithelial monolayer, if an epithelial node moves towards the lumen, then the local signed curvature will be negative and the basement membrane force will act to pull the node towards the tissue stroma. Conversely, if an epithelial cell moves towards the stroma, then the local signed curvature will be positive and it will be pushed away by the same force.

For the purposes of this model, it is necessary to approximate Eq. (4) to calculate the discrete curvature at a point $\left(x_{i}, y_{i}\right)=\left(x\left(s_{i}\right), y\left(s_{i}\right)\right)$ on a piecewise linear curve defined using the two neighbouring points, $\left(x_{i-1}, y_{i-1}\right)$ and $\left(x_{i+1}, y_{i+1}\right)$. Now,

$\left(\Delta s_{i}\right)^{2}=\left(x_{i}-x_{i-1}\right)^{2}+\left(y_{i}-y_{i-1}\right)^{2}$,

and the positive square root is taken so that

$s_{i}=\sum_{j=0}^{i} \Delta s_{j}$

is strictly increasing. The first and second derivatives are approximated using central differences (Süli and Mayers, 2003) so that

$x^{\prime}\left(s_{i}\right) \approx \frac{x_{i+1}-x_{i-1}}{\Delta s_{i}+\Delta s_{i+1}}$,

$x^{\prime \prime}\left(s_{i}\right) \approx 2\left[\frac{\Delta s_{i} x_{i+1}-\left(\Delta s_{i}+\Delta s_{i+1}\right) x_{i}+\Delta s_{i+1} x_{i-1}}{\Delta s_{i} \Delta s_{i+1}\left(\Delta s_{i}+\Delta s_{i+1}\right)}\right]$,

and $y^{\prime}\left(s_{i}\right)$ and $y^{\prime \prime}\left(s_{i}\right)$ are found similarly.

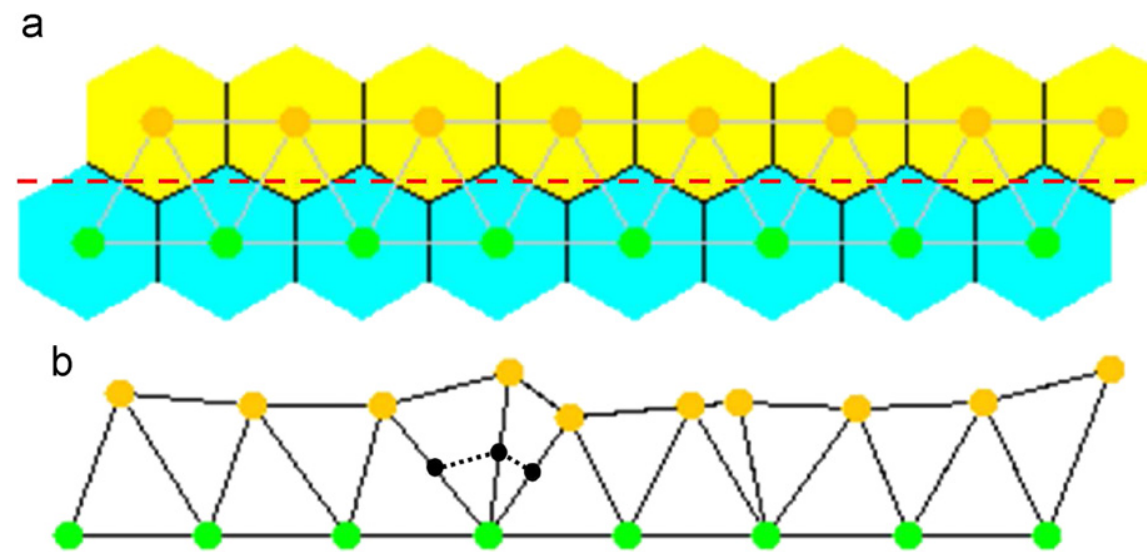

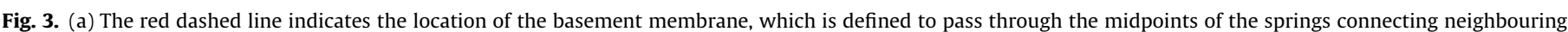

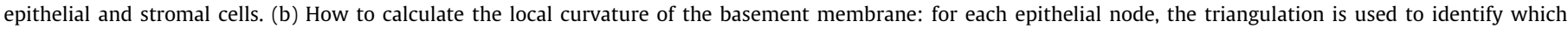

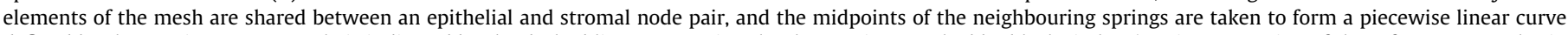

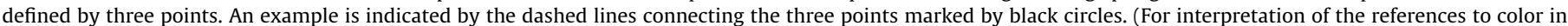
this figure legend, the reader is referred to the web version of this article.) 
These approximations are applied to Eq. (4) to evaluate the discrete curvature at a particular spring midpoint. The discrete curvature is then applied to calculate the restoring force that is applied to each individual epithelial node $i$, subject to each cell $j$ in the set of neighbouring stromal cells, $N_{i}$ :

$\mathbf{F}_{i}=\beta \sum_{\forall j \in N_{i}} \kappa_{i j} \hat{\mathbf{u}}_{i, j}$

where $\beta$ is the basement membrane force parameter, which characterises the strength of adhesion of the epithelial layer to the basement membrane, and the stiffness of the membrane itself, $\hat{\mathbf{u}}_{i j}$ is the unit vector from the stromal node $j$ to the epithelial node $i$, and $\kappa_{i j}$ is the calculated local discrete curvature for that cell pair.

\subsection{Chaste}

Simulations and model development are conducted within the Cancer, Heart And Soft Tissue Environment (Chaste) framework, an open source software library written in object-oriented $\mathrm{C}++$ and constructed using agile programming. Chaste is a general purpose simulation package aimed at multi-scale, computationally demanding problems arising in biology and physiology, developed using agile programming techniques and following software engineering practices. Released under the LGPL 2.1 open source license, it is available as a resource to the research community (http://www.cs.ox.ac.uk/chaste) (Pitt-Francis et al., 2009).

Simulations that are described in Section 3 are conducted within a graphics visualisation tool created purposely in Java for Chaste, and a typical simulation of 100 cell hours requires approximately $2.8 \mathrm{~min}$ of CPU time on a desktop Linux PC (u.c.) with an Athlon 5200B processor. The code that is used to run these simulations is released and thus available to download from the Chaste website.

\section{Simulation results}

Results from simulations of the model described in Section 2 are now presented and discussed. Fig. 1 illustrates the typical initial configuration of cells that is used for the experiments and, unless stated otherwise, the parameters used are provided in Table 1 . The distances in the model have been scaled with cell width $(10 \mu \mathrm{m}$ Alberts et al., 2002; Smallwood, 2009), time is measured in hours, and Eqs. (1) and (2) have been scaled so that $\eta=1$ (Meineke et al., 2001; Van Leeuwen et al., 2009). All other variables have been scaled accordingly. The investigations presented here focus on the role of the basement membrane force, and whether this is affected by the strength of adhesive and repulsive forces between cells. The aim of this work is to determine the parameters required to maintain a stable configuration of cells, in order to achieve a realistic model of healthy cell division and migration within the epithelial monolayer. Subsequently it is possible to examine under which conditions the layer will buckle, and what drives this process. For the following results, unless stated otherwise, all springs in the model have been assigned equal strength.

To produce plots that are indicative of the general behaviour of the model under different conditions, 100 simulations were run for each set of parameters investigated, and the average results taken. Each simulation is run for a duration of $125 \mathrm{~h}$, and the results are calculated at the final timestep. This ensures that a number of divisions will have occurred for each cell and enough simulations are run to produce results that are indicative of the general behaviour of the system. Simulation movies that correspond to the different cases examined here are contained within Supplementary material.

The stochastic nature of the model is due to the cell division events in the proliferating layer, which are determined using the cell cycle model. The pseudo-random number generator used is re-seeded for each simulation to generate a new, independent set of birth events. More specifically, the seed used is unique to the simulation index from 0 to 99, and therefore 100 independent sequences of birth events are chosen and used throughout. This enables identical experiments to be run when varying the parameter of interest, thereby allowing accurate comparison of behaviour for different parameter values.

The ideal state of the layer of proliferating cells is to be flat, given the geometry applied here, this corresponds to the locus of the epithelial cell centres lying parallel to the $x$-axis. The flatness of the layer is described by the metric $\alpha$, measured as the average gradient of the lines connecting neighbouring cell pairs (the average gap ratio). That is, the modulus of the vertical and horizontal distance between each pair of neighbouring proliferating cell centres is found, the individual ratios calculated and then averaged over all the pairs. So for $n$ cells in the layer, moving in order of increasing $x$-coordinate, $\alpha$ is given by

$\alpha=\frac{1}{(n-1)} \sum_{i=1}^{n-1}\left|\frac{y_{i+1}-y_{i}}{x_{i+1}-x_{i}}\right|$

This gives an indication of how wrinkled the layer is, such that the layer is described as flat if $\alpha \approx 0$, and ensures that no cancelling occurs between consecutive positive and negative gradients.

\subsection{Increasing the basement membrane force}

Firstly, results are presented that examine the effect of increasing the basement membrane force when the spring strength for the interactive forces between all cells are equal, and hence assigned $\mu=15$ as in Table 1 . Given that the value of the basement membrane force changes for each cell at each timestep, dependent on the local curvature, these plots indicate changes with $\beta$. To emphasise the quantitative behaviour of this system, the value of this force is typically one quarter of the magnitude of the linear spring forces acting between cells.

Fig. 4(a) plots the average horizontal (solid line) and vertical (dashed line) distance between neighbouring pairs of epithelial cells against $\beta$, which are combined to show the change in the average gap ratio, $\alpha$, in Fig. 4(b). Note that these distances are

Table 1

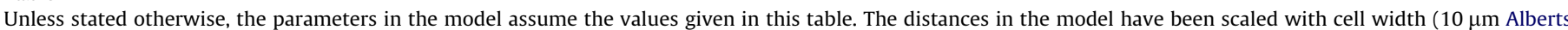
et al., 2002, Smallwood, 2009), time is measured in hours, and all other variables are nondimensionalised accordingly.

\begin{tabular}{|c|c|c|c|c|}
\hline Parameter & Description & Value & Units & Reference \\
\hline$\mu$ & Spring strength & 15 & $\mathrm{~N}$ Cell width ${ }^{-1}$ & Meineke et al. (2001) \\
\hline$\eta$ & Constant drag coefficient & 1 & N hours Cell width ${ }^{-1}$ & Meineke et al. (2001) \\
\hline$s$ & Equilibrium spring rest length & 1 & Cell width & Van Leeuwen et al. (2009) \\
\hline$\Delta t$ & Timestep & 0.0042 & Hours & \\
\hline$\beta$ & Basement membrane force parameter & See text & N Cell width ${ }^{-1}$ & \\
\hline
\end{tabular}


a

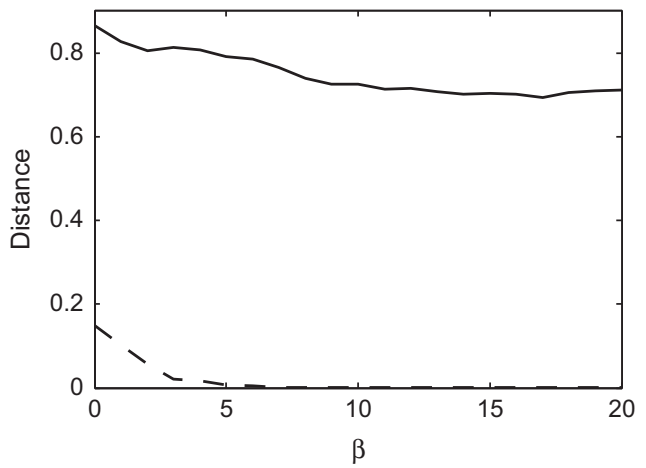

b

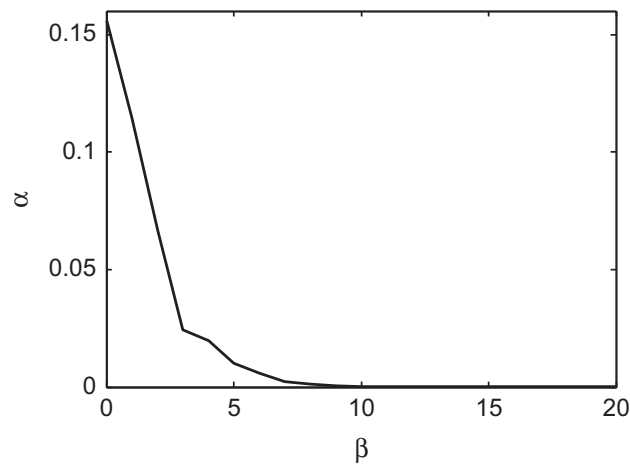

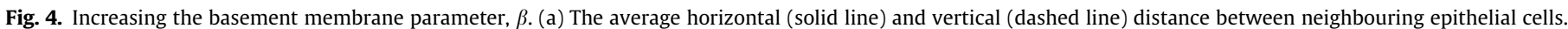

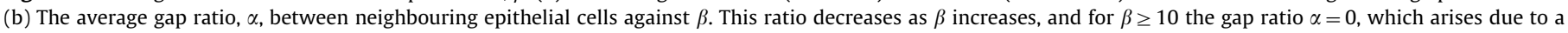
zero vertical distance between neighbouring epithelial cells and hence the layer is completely flat.

a

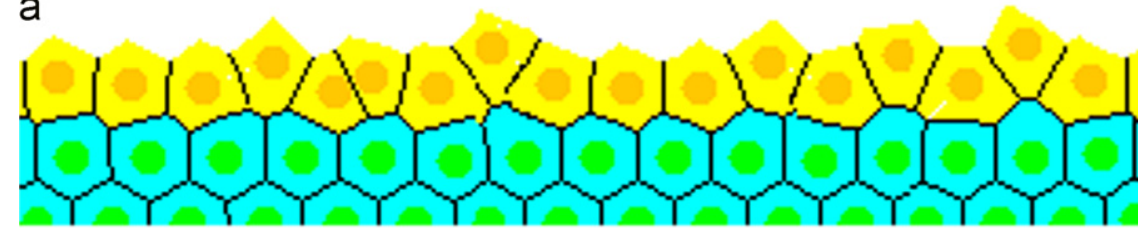

$\mathrm{b}$

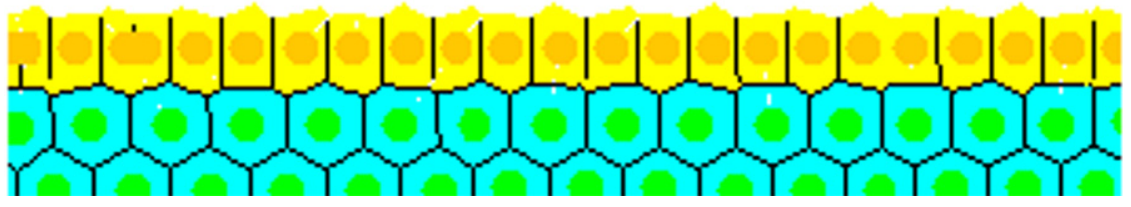

Fig. 5. Snapshots taken from simulations to show the typical behaviour for (a) $\beta=0$, (b) $\beta=15$.
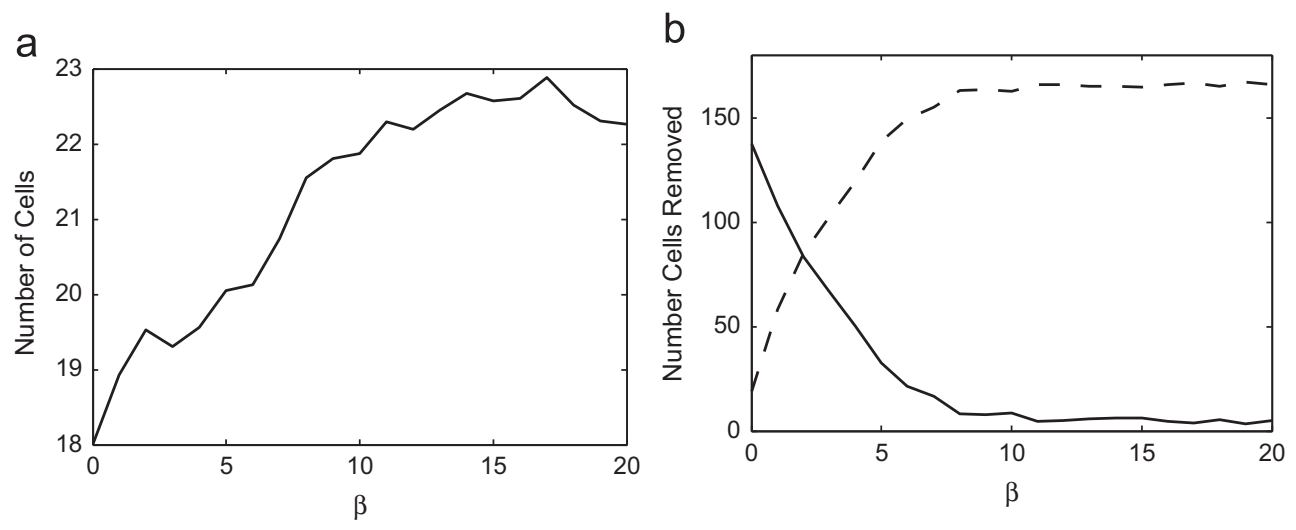

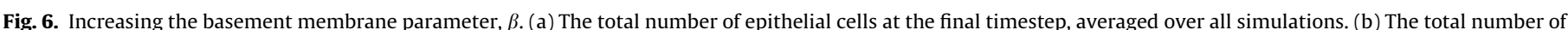

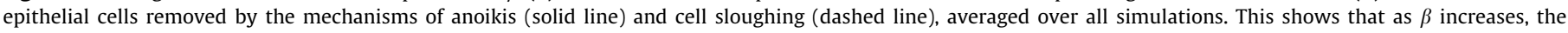

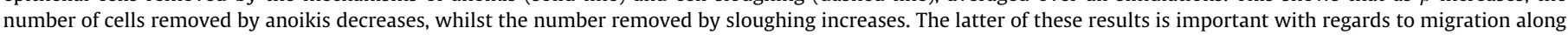
the epithelial layer.

scaled with the equilibrium cell width and hence are nondimensional. These plots show that as $\beta$ increases, both the horizontal and vertical distances between neighbouring epithelial cell centres decrease. The consequence of this is clearly seen in the decrease of $\alpha$, which is zero for $\beta \geq 10$, and hence the layer is flat when a sufficiently high basement membrane force is applied.

Fig. 5 illustrates this behaviour by comparing two snapshots from simulations: Fig. 5(a) corresponds to $\beta=0$ and Fig. 5(b) corresponds to $\beta=15$. These snapshots show that the layer is completely flat for $\beta=15$, whereas it is wrinkled and cells are being forced out of the layer when $\beta=0$, eventually undergoing apoptosis by anoikis. Simulation Movies 1 and 2, which correspond to these two cases, are also provided in the supplementary material and illustrate the contrasting behaviour.

Supplementary material related to this article can be found online at doi:10.1016/j.jtbi.2011.12.013.

Fig. 6(a) plots the total number of epithelial cells in the monolayer, which increases as $\beta$ increases. This is in accordance with the decreasing horizontal gap between neighbouring cells shown in Fig. 4(a). Fig. 6(b) plots the total number of cells removed both by anoikis and by sloughing at the edges over the course of the simulation. In the absence of the force due to the 
basement membrane, the proliferating epithelial cells are quickly and easily pushed upwards, losing contact with the stromal cells below, and so are removed by anoikis. However, as $\beta$ increases, this total decreases from approximately 140 to less than 10 . This result shows that the basement membrane force plays a role in preventing detachment of the epithelial cells by correcting the bending in the layer that leads to cells moving upwards.

The opposite behaviour is observed for the number of cells that are removed by sloughing at the edges-this total increases from approximately 20 to 160 . This reveals a marked change in the dynamics of the system, whereby the extent of cell migration increases as $\beta$ increases, rather than cells being simply forced upwards and out. Thus it is demonstrated that the basement membrane force plays a key role in the model, to favour horizontal migration of the proliferating cells, and to prevent cell detachment from the stromal layer by correcting unwanted bending. Together with Fig. 4 these results indicate that a basement membrane force parameter of $\beta \geq 10$ is required to maintain such a stable, flat layer whilst epithelial cells migrate and few cells are removed by anoikis.

\subsection{Varying epithelial cell adhesion}

While the layer remains flat for $\beta \geq 10$, the average horizontal distance between epithelial cells decreases as $\beta$ increases, so that the cells are positioned closer together. For the cells to move towards a state of equilibrium the springs should be at, or tending to, the natural rest length, which is 1 cell width. It is therefore important to investigate the effect of varying the spring strength parameters, to ascertain if it is possible to improve on these results.

For a fixed value of $\beta=12$, the effect of increasing the ratio of spring strengths between neighbouring epithelial cells (E-E) to neighbouring stromal cells (S-S) is investigated. Fig. 7(a) shows the variation in the average horizontal and vertical distance between neighbouring epithelial cells, Fig. 7(b) how this is realised in the ratio $\alpha$, Fig. 7(c) the total number of epithelial cells and Fig. 7(d), how many are removed by anoikis and sloughing. These results can be compared with those in Figs. 4 and 6.

Firstly, from Fig. 7(a), the horizontal distance between cells increases towards 1 as the relative strength of the E-E springs increases, and the vertical distance is unchanged. This coincides with the total number of cells in the layer decreasing in Fig. 7(c). As shown in Fig. 7(b), when the strength of the E-E springs is weaker than that of the S-S springs (the ratio is less than 1 ), the average gap ratio $\alpha$ is larger than that for when the E-E springs are stronger-this is a consequence of the increasing horizontal distance between cells, which arises due to the stronger repulsive force when cells are under compression. As the E-E spring strength increases further, the change in $\alpha$ is negligible.

However, given the results in Fig. 7(d), when the E-E spring strengths increase beyond the $\mathrm{S}-\mathrm{S}$ spring strengths to the extent that the springs are tending to the natural spring length, the trend shows that sloughing is decreased but anoikis is increased. Hence, too strong adhesion/repulsion between epithelial cells reduces migration. Despite this, the results suggest that increasing the spring strength between pairs of epithelial cells to a factor of 2.5 times that of other springs will yield cells with natural sizes (such that the spring lengths are close to the equilibrium value), without overly compromising the extent of cell migration. Simulation Movie 3, provided in Supplementary material, is an example of a typical simulation where $\beta=12$ and the E-E/S-S spring strength ratio is 2.5 .

Supplementary material related to this article can be found online at doi:10.1016/j.jtbi.2011.12.013.

To demonstrate that these results are consistent for increasing values of $\beta$, Fig. 8 compares the total number of epithelial cells in

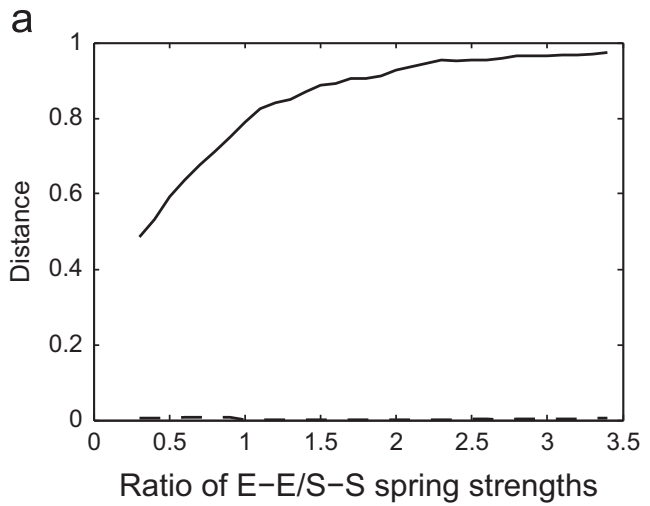

b
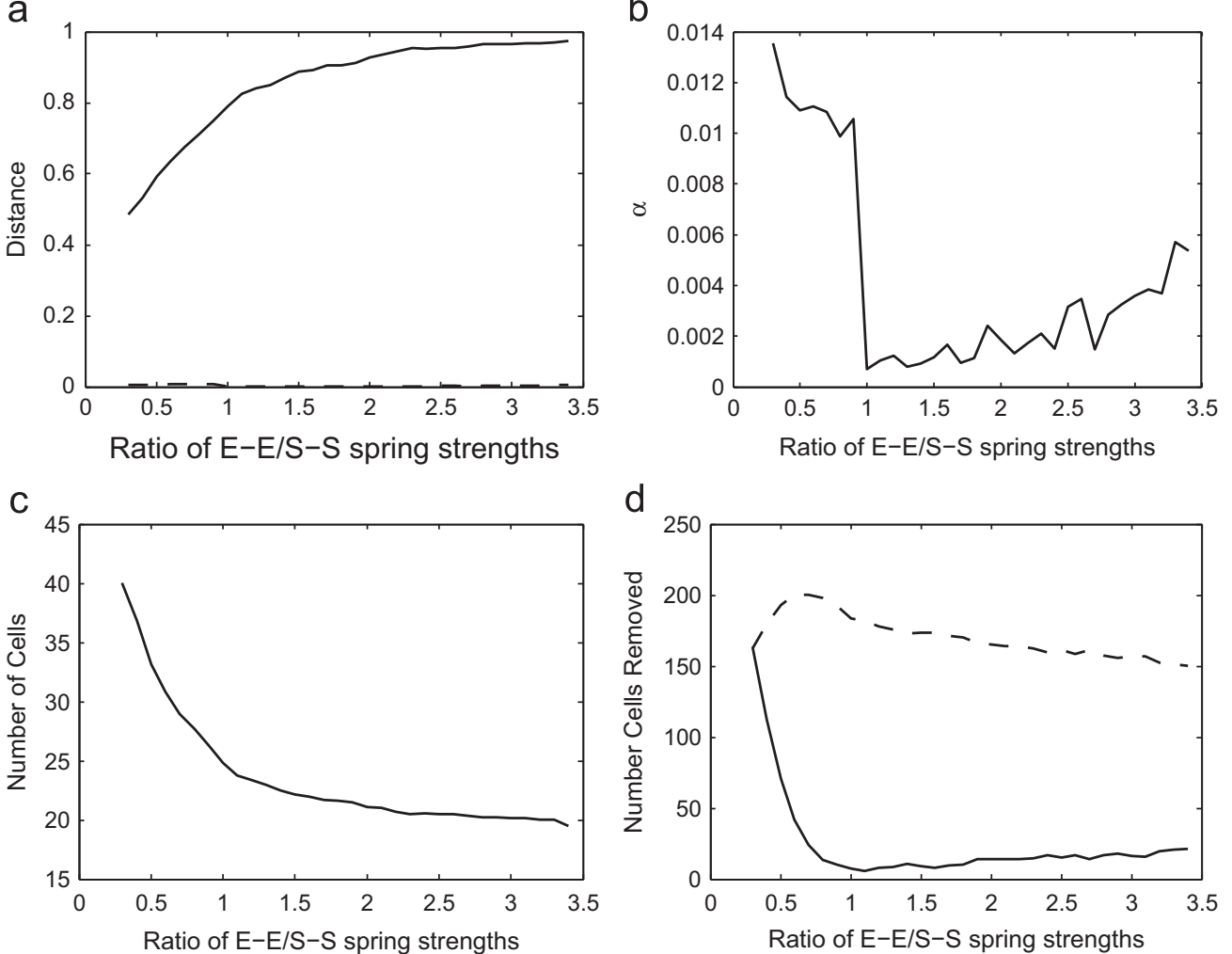

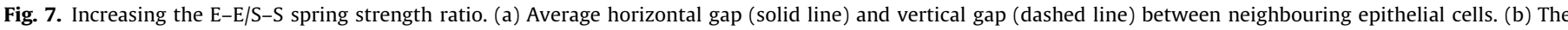

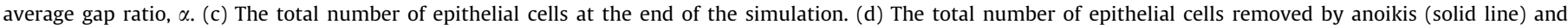
sloughing (dashed line) at the edges. 
the layer at the final timestep $(t=125 \mathrm{~h})$ as the E-E/S-S spring strength ratio increases, for $\beta=8,12,16$. This plot shows that the difference for each value of $\beta$ is negligible. Further investigations show that changing the ratio of spring strengths between neighbouring epithelial cells and those connecting epithelial and stromal cells (E-S/E-E) has negligible effect on the dynamics of cell movement, and so these results are omitted for brevity. This occurs because the E-S springs only exert a force under repulsion.

\subsection{Hyperproliferation}

An increase in proliferative capacity is a known hallmark of cancerous cells (Hanahan and Weinberg, 2000). Indeed, in CRC, the inactivation of the APC tumour suppressor gene is one of the ratelimiting and initiating events (Boman et al., 2008; Fodde and

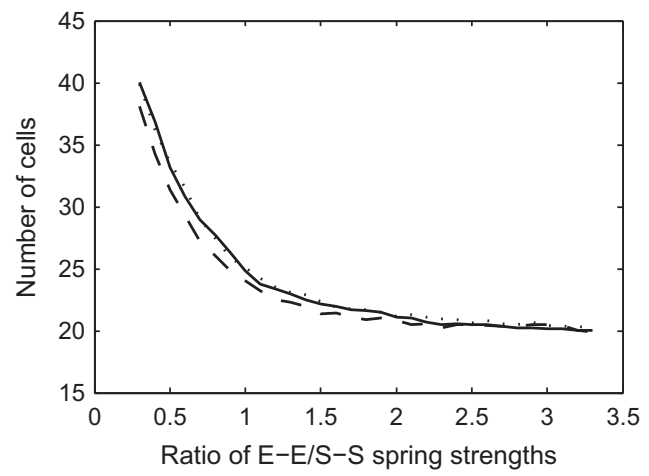

Fig. 8. Increasing the E-E/S-S spring strength ratio and comparing the total number of epithelial cells at the end of the simulation for increasing values of $\beta$ : $\beta=8$ (dashed line), $\beta=12$ (solid line), $\beta=16$ (dotted line).

a

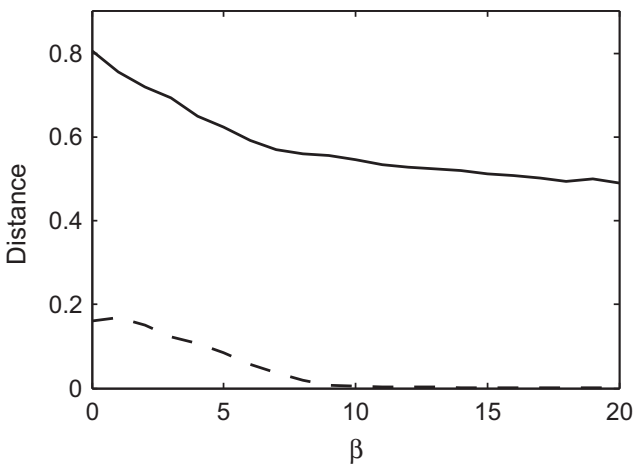

C

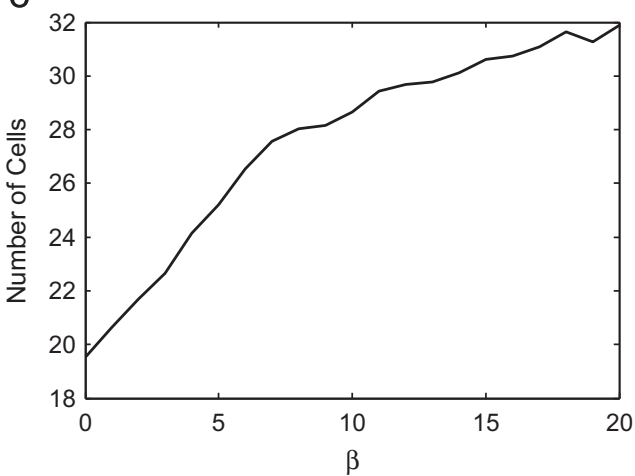

Brabletz, 2007; Humphries and Wright, 2008). One downstream effect of the loss of APC is an increase in proliferation, either through an increased proliferative capacity for individual cells, or an increased proliferative compartment (Näthke, 2004; Sansom et al., 2004). By reducing the cell cycle time for each cell in the monolayer, it is possible to investigate hyperproliferation in this model and observe the effects on the structure. Fig. 9 plots the corresponding results to Figs. 4 and 6, where the duration of each phase of the cell cycle is reduced, now chosen from a $\mathrm{U}(3,5)$ distribution.

Fig. 9(a) shows that the horizontal distance separating neighbouring cells decreases as in Fig. 4(a), but the decrease is greater under these new conditions, such that the cells are more closely packed in the monolayer and the average horizontal separation is approximately 0.5 for $\beta \geq 10$. Accordingly, the total number of epithelial cells in the layer increases to over 30, as shown in Fig. 9(c). The vertical separation also decreases, but at a slower rate than under normal conditions. When these results are combined in Fig. 9(b), $\alpha$ does not decrease as sharply, and does not reach zero as before. This implies that the layer is wrinkled, and never attains a purely flat state with this chosen rate of proliferation.

Fig. 9(d) shows a major difference in the number of cells removed by anoikis and by sloughing - in each case the number of cells removed by each method has increased greatly from simulations run under normal conditions. The total number of cells removed by anoikis decreases from approximately 400 to 350 as $\beta$ increases, while those removed by sloughing increases from approximately 50 to 450 . A switch in behaviour has occurred; before, fewer cells were removed by anoikis as $\beta$ increased. Now it is the case that this total levels out at a much higher value, so that both cell removal mechanisms are employed as the layer seeks to attain a state of equilibrium. This behaviour is due to the

b

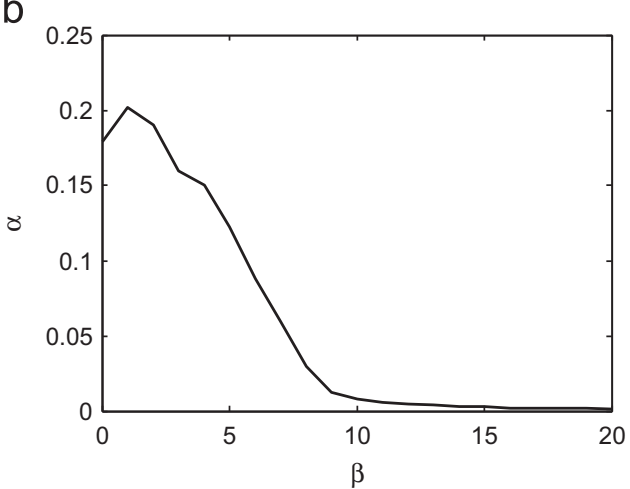

d

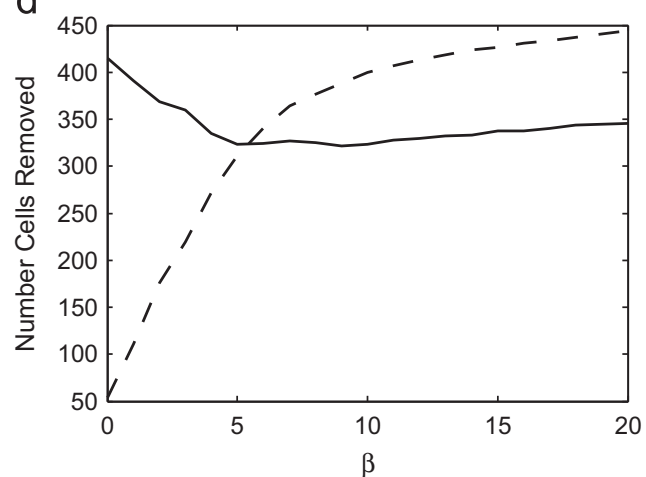

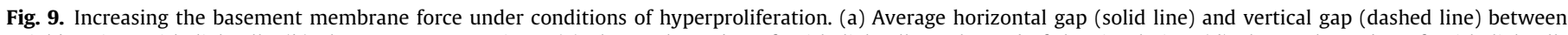

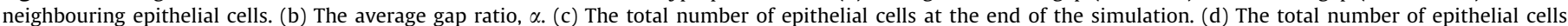
removed by anoikis (solid line) and sloughing (dashed line) at the edges. 
total number of cells in the layer, which increases with $\beta$ and therefore leads to more birth events, and subsequently a greater number of cells that must be removed.

Simulation Movie 4, provided in Supplementary material, shows a typical example of the evolution of the layer under hyperproliferative conditions, where $\beta=12$ and the E-E/S-S spring strength ratio is 2.5 . This can be compared directly with Movie 3 , the case where individual cell cycle times are chosen from a $U(11,13)$ distribution. Movie 4 illustrates how hyperproliferation induces local buckling of the layer.

Supplementary material related to this article can be found online at doi:10.1016/j.jtbi.2011.12.013.

From these results, it is demonstrated that a simple increase in the rate of cell division within the epithelial monolayer does not simply lead to an increase in migration, but rather increases the vulnerability of the layer to buckling. This is clear as anoikis is required to a much greater extent than for normal cell division events. Consequently, should excessive cell division be coupled with failed apoptosis cues, then hypertrophy would result, disrupting the tissue architecture.

\section{Discussion}

The aim of this model is to incorporate the role of the basement membrane in a simple geometric representation of a proliferating layer of epithelial cells constrained to lie on a bed of stromal cells, and under healthy conditions for this monolayer to demonstrate cell division that prompts in-plane migration whilst remaining flat. The components of the model outlined in Section 2 have been investigated, and the following behaviour observed, which enables regions of validity for the model to be determined.

The results show that the basement membrane force is crucial in maintaining the stability of the monolayer of proliferating cells. As expected, the larger this force is, as controlled by the value of the parameter $\beta$, the flatter the layer remains, as defined by $\alpha$, and fewer cells are forced to detach from the underlying stroma. This permits the use of anoikis as a complementary control mechanism for the system. Specifically, results show that $\alpha \approx 0$ for $\beta \geq 10$, and hence this is sufficient to keep the monolayer flat and prevent wrinkling.

In conjunction with the basement membrane force, the strength of the interactive forces between neighbouring proliferative cells plays a role in controlling the inter-cell spacing-these forces combine the attraction caused by adhesion molecules in the cell membranes, and the repulsion caused by the limited compressibility of each cell. The Delaunay triangulation, and the implementation of anoikis, ensures that epithelial cells retain connections to the basement membrane, as well as to their immediate neighbours. The results show that for high values of the basement membrane force parameters, stronger forces between neighbouring cells are required to prevent overcrowding, and under these conditions the monolayer tends towards a state of equilibrium. There exists, however, a limit to this spring strength above which a higher number of cells are removed by anoikis, and the rate of cell migration decreases. Specifically, the results show that to prevent this and to maintain homeostatic dynamics, the E-E spring strength should be less than or equal to 2.5 for a basement membrane parameter value $\beta=12$. The high basement membrane parameter ensures that bending is counteracted to keep the layer flat, while the strength of the forces between neighbouring epithelial cells is increased enough to prevent overcrowding.

A simple experiment to investigate the consequences of increased cell proliferation shows that the layer is more likely to be corrupted, as $\alpha$ does not decrease as sharply and reach zero as under normal proliferative rates. This implies that the layer is wrinkled, and never attains a purely flat state with this chosen rate of proliferation (as shown in the simulation Movie 4, provided in Supplementary material). Results also show that both cell removal mechanisms are employed as the layer seeks to attain a state of equilibrium, and this is especially important in light of failed apoptotic cues that can characterise cancerous cells, prolonging survival in the absence of matrix attachment (Grossman, 2002).

In this current form, the model is sufficiently general to be applied to a range of biological epithelia. One specific application that has been referred to is that of the colonic crypt. A natural extension of this work is to adopt the configuration of the crosssection of the crypt embedded within the surrounding tissue stroma, and so extend this model to account for the role of the basement membrane at the semi-spherical base of the crypt. In this context, different epithelial cell types can be distinguished such that more realistic cell cycle models can be implemented, incorporating cell signalling factors that are dependent on cell location within the crypt. Further, heterogeneities in the connective tissue can be explored, in light of contributing factors to the behaviour and decisions of the epithelial cells.

\section{Acknowledgements}

S.J.D. is supported by an EPSRC-funded Life Sciences Interface Doctoral Training Centre studentship (Grant reference EP/ E501605/1). A.G.F., D.J.G. and J.M.O. are funded through the OCISB project (BB/D020190/1).

\section{References}

Alberts, B., Johnson, A., Lewis, J., Raff, M., Roberts, K., Walter, P., 2002. Molecular Biology of the Cell, fourth ed. Garland Science, New York.

Ascher, U., Petzold, L., 1998. Computer Methods for Ordinary Differential Equations and Differential Algebraic Equations. SIAM, Philadelphia.

Boman, B.M., Fields, J.Z., Cavanaugh, K.L., Guetter, A., Runquist, O.A., 2008. How dysregulated colonic crypt dynamics cause stem cell overpopulation and initiate colon cancer. Cancer Res. 63, 3304-3313.

Bullen, T.T., Forrest, S., Campbell, F., Dodson, A.R., Hershman, M.J., Pritchard, D.M., Turner, J.R., Montrose, M.H., Watson, A.J.M., 2006. Characterization of epithelial cell shedding from human small intestine. Lab. Invest. 86, 1052-1063.

Clayton, E., Doupé, D.P., Klein, A.M., Winton, D.J., Simons, B.D., Jones, P.H., 2007. A single type of progenitor cell maintains normal epidermis. Nature 446, 185-189.

Drasdo, D., Loeffler, M., 2001. Individual-based models to growth and folding in one-layered tissues: intestinal crypts and early development. Nonlinear Anal. 47, 245-256.

Duggan, C.D., Ngai, J., 2007. Scent of a stem cell. Nat. Neurosci. 10, 673-674.

Edwards, C.M., Chapman, S.J., 2007. Biomechanical modelling of colorectal crypt budding and fission. Bull. Math. Biol. 69, 1927-1942.

Fleming, E.S., Zajac, M., Moschenross, D.M., Montrose, D.C., Rosenberg, D.W Cowan, A.E., Tirnauer, J.S., 2007. Planar spindle orientation and asymmetric cytokinesis in the mouse small intestine. J. Histochem. Cytochem. 55, $1173-1180$.

Fodde, R., Brabletz, T., 2007. Wnt $/ \beta$-catenin signalling in cancer stemness and malignant behaviour. Curr. Opin. Cell Biol. 19, 150-158.

Frisch, S.M., Francis, H., 1994. Disruption of epithelial cell-matrix interaction induces apoptosis. J. Cell. Biol. 124, 619-626.

Grossman, J., 2002. Molecular mechanisms of "detachment-induced apoptosis-Anoikis". Apoptosis 7, 247-260.

Hanahan, D., Weinberg, R.A., 2000. The hallmarks of cancer. Cell 100, 57-70.

Honda, H., 1983. Geometrical models for cells in tissues. Int. Rev. Cystol. 81 $191-248$.

Honda, H., Tanemura, M., Nagai, T., 2004. A three-dimensional vertex dynamics cell model of space-filling polyhedra simulating cell behaviour in a cell aggregate. J. Theor. Biol. 226, 439-453.

Humphries, A., Wright, N., 2008. Colonic crypt organization and tumorigenesis Nat. Rev. Cancer 8, 415-424.

Loeffler, M., Potten, C.S., Paulus, U., Glatzer, J., Chwalinkski, S., 1988. Intestinal cell proliferation II. Computer modelling of mitotic index data provides further evidence for lateral and vertical cell migration in the absence of mitotic activity. Cell Tissue Kinet. 21, 247-258.

Meineke, F.A., Potten, C.S., Loeffler, M., 2001. Cell migration and organization in the intestinal crypt using a lattice-free model. Cell Prolif. 34, 253-266.

Näthke, I., 2004. The adenomatous polyposis coli protein: the achilles heel of the gut epithelium. Annu. Rev. Cell Dev. Biol. 20, 337-366. 
Pitt-Francis, J., Pathmanathan, P., Bernabeu, M.O., Bordas, R., Cooper, J., Fletcher, A.G., Mirams, G.R., Murray, P., Osborne, J.M., Walter, A., Chapman, S.J., Garny, A., van Leeuwen, I., Maini, P.K., Rodrguez, B., Waters, S.L., Whiteley, J.P., Byrne, H.M., Gavaghan, D.G., 2009. Chaste: a test-driven approach to software development for biological modelling. Comput. Phys. Commun. 180 2452-2471.

Sansom, O.J., Reed, K.R., Hayes, A.J., Ireland, H., Brinkmann, H., Newton, I.P., Batlle E., Simon-Assmann, P., Clevers, H., Näthke, I.S., Clarke, A.R., Winton, D.J., 2004 Loss of Apc in vivo immediately perturbs Wnt signaling, differentiation, and migration. Gene Dev. 18, 1385-1390.

Savla, U., Olson, L.E., Waters, C., 2004. Mathematical modeling of airway epithelial wound closure during cyclic mechanical strain. J. Appl. Physiol. 96, 566-574.

Shanmugathasan, M., Jothy, S., 2000. Apoptosis, anoikis and their relevance to the pathobiology of colon cancer. Path. Int. 50, 273-279.
Smallwood, R., 2009. Computational modelling of epithelial tissues. WIREs Syst. Biol. Med. 1, 191-201.

Süli, E., Mayers, D., 2003. An Introduction to Numerical Analysis. Cambridge University Press.

Swat, M., Kel, A., Herzel, H., 2004. Bifurcation analysis of the regulatory modules of the mammalian $G_{1} / S$ transition. Bioinformatics 20, 1506-1511.

Trier, J.S., Allan, C.H., Abrahamson, D.R., Hagen, S.J., 1990. Epithelial basement membrane of mouse jejunum. J. Clin. Invest. 86, 87-95.

Van Leeuwen, I.M.M., Mirams, G.R., Walter, A., Fletcher, A., Murray, P., Osborne, J., Varma, S., Young, S.J., Cooper, J., Pitt-Francis, J., Momtahan, L., Pathmanathan, P., Whiteley, J.P., Chapman, S.J., Gavaghan, D.J., Jensen, O.E., King, J.R., Maini, P.K., Waters, S.L., Bryne, H.M., 2009. An integrative computational model for intestinal tissue renewal. Cell Prolif. 42, 617-636.

Wright, N., Alison, M., 1984. The Biology of Epithelial Cell Populations. Oxford University Press, Oxford. 